\title{
MÉTODO FUZZY PARA CLASSIFICAÇÃO DE PORTFÓLIO DE COMPRAS
}

\author{
Aníbal Alberto Vilcapoma Ignacio \\ Universidade Federal Fluminense/UFF \\ Campus de Petrópolis/RJ \\ anibalvilcapoma@gmail.com \\ Lea Maria Dantas Sampaio \\ Ecce Solutions Ltda. \\ Rio de Janeiro/RJ \\ leasampaio@gmail.com
}

\begin{abstract}
RESUMO
As atividades de compras vêm ganhando importância, passando a ser tratada como estratégica, dentro das organizações. O presente artigo apresenta uma metodologia de classificação de materiais, que usa os critérios de criticidade e complexidade. A metodologia utiliza o processo de inferência com os conjuntos fuzzy para a classificação dos itens de compra de materiais agrupados. Resultados computacionais são apresentados, de modo a exemplificarem a eficácia da dita metodologia.
\end{abstract}

Palavra-chave: Lógica fuzzy; Matriz de portfólio de compras; Metodologia de classificação de materiais.

\begin{abstract}
Purchasing activities have been gaining importance and have been treated as strategic within organizations. This paper presents a material classification methodology that uses the criteria of criticality and complexity. The methodology uses the inference process with fuzzy sets for the classification of grouped material purchase items. Computational results are presented to exemplify the effectiveness of said methodology.
\end{abstract}

Keywords: Fuzzy logic; Purchasing portfolio matrix; Material classification methodology.

\section{INTRODUÇÃO}

Ao longo das últimas décadas, a atividade de compras vem ganhando importância, passando a ser tratada como estratégica, dentro das organizações. A literatura pertinente aponta que a maneira como uma empresa se relaciona com seus fornecedores, na cadeia de suprimentos, pode ser um fator competitivo importante e constituir um diferencial estratégico nos mercados que atua [1] e [2]. Nesse contexto, a Matriz de Portfólio de Compras (MPC) é uma ferramenta relevante, pois auxilia na elaboração de estratégias capazes de minimizar os riscos no suprimento e potencializar o poder de compra das organizações. A MPC ganhou crescente reconhecimento e passou a ser largamente utilizada por profissionais da atividade de compras, especialmente nos EUA, Canadá e Europa. 
Contudo, as organizações de países em desenvolvimento, como a Índia e o Brasil, encontram-se incipientes com relação à adoção da MPC.

O presente artigo apresenta uma metodologia de classificação de materiais, usando os critérios de criticidade e complexidade. A metodologia utiliza o processo de inferência dos conjuntos fuzzy como método de classificação. Resultados computacionais são apresentados, de modo a exemplificar a dita metodologia.

O artigo apresenta a seguinte estrutura: introdução do artigo; uma revisão dos modelos de portfólio de compras e de lógica fuzzy; exemplo de modelo de classificação aplicado em empresa pública, que utiliza a lógica fuzzy; os resultados computacionais do modelo-exemplo. Finalmente, apresenta-se as considerações finais.

\section{REVISÃO DA LITERATURA SOBRE PORTFÓLIO E LÓGICA FUZZY}

Segundo [3], a teoria de portfólios é proposta em [4], no contexto da gestão de investimentos em títulos imobiliários, com o objetivo de maximizar a rentabilidade para um dado risco ou minimizar o risco para uma determinada rentabilidade. Desde então, a literatura registrou a utilização crescente de modelos de portfólio nas mais variadas aplicações [5]. A Matriz BCG (Boston Consulting Group), que está entre os modelos mais conhecidos e utilizados no meio empresarial, propõe que os produtos ou unidades de negócios de uma determinada empresa sejam classificados em duas dimensões: a taxa de crescimento do mercado e a participação de mercado. A proposição básica dos modelos de portfólios é que situações diferentes requerem estratégias distintas [6]. Nos últimos 20 anos, um grande número de modelos de portfólio foi proposto - tendo ora os produtos comprados, ora a relação entre fornecedores e compradores como unidade de análise.

No que tange à lógica fuzzy ou nebulosa, é fundamental a noção da teoria dos conjuntos fuzzy, proposta por [7] e que apresenta um tratamento formal e objetivo do processo de tomada de decisão em ambientes nebulosos, ou seja, com informações imprecisas e difusas. Sua base de conhecimento sobre um problema pode ser definida em termos linguísticos. Isto é relevante pois, à medida que a complexidade de um sistema aumenta, a capacidade humana de descrevê-lo de forma precisa e clara diminui [8].

\subsection{PORTFOLIO DE COMPRAS}

A literatura registra a aplicação dos modelos de portfólio de compras em diversos contextos empresariais. Em [9] descreve-se a aplicação do portfólio de compras no setor de construção civil, em uma multinacional que possui projetos atuantes no mercado de dois países com ambientes muito distintos: Portugal e Angola. Em [10] propõe-se um modelo, aplicado à indústria automotiva, que avalia a influência dos fornecedores nas especificações técnicas das categorias avaliadas de produtos, indicando o grau de dependência do fabricante, junto a seus fornecedores, e propondo estratégias de relacionamento específicas para cada caso. Em [11] desenvolve-se um modelo de portfólio para analisar os aspectos de poder e interdependência nas relações entre compradores e fornecedores. Em [12] descrevese a aplicação do portfólio de compras em uma empresa da indústria do petróleo que atua em um local remoto, propondo adaptações ao modelo devido às dificuldades de infraestrutura e logística enfrentadas pela empresa. Em [13] propõe-se critérios específicos para avaliar o risco de suprimento de uma empresa fabricante de refrigeradores, utilizando-se números fuzzy para medir os critérios qualitativos. Em [14] propõe-se uma taxonomia para diferenciar estratégias de compras em empresas industriais.

No âmbito da gestão da atividade de compras, os modelos de portfólio podem auxiliar a alocação de recursos críticos ao identificar os produtos/serviços e fornecedores que requerem maior atenção [15]. Em [16] alertam que, apesar do número crescente de estudos utilizando a Matriz de Portfólio de Kraljic (MPK), faltam orientações claras sobre: (i) como 
devem ser posicionadas as categorias de produtos na matriz; (ii) como, especificamente, são desenvolvidas as estratégias de suprimento e (iii) quais os resultados obtidos com a utilização de modelos de portfólio na atividade de compra. Os autores ressaltam que não há regras exatas para a classificação de produtos e suas categorias, na matriz de portfólio, e que as avaliações dos itens nas duas dimensões da matriz carregam subjetividade e imprecisão. Em [15], ressalta-se que a etapa de classificação e segmentação dos produtos na matriz de portfólio, apesar de ser altamente subjetiva, é a etapa mais importante da metodologia pois, ao elaborarem o modelo de portfólio, os tomadores de decisão devem argumentar e chegar a um consenso sobre a importância relativa de cada critério que compõe as dimensões.

Em [6], afirmam que um ponto essencial na construção dos modelos de portfólio é a correta definição dos critérios de segmentação das compras. Em [17], o autor considera as formas de medição e posicionamento como um ponto fraco de todos os modelos de portfólio. Em [18], conclui-se que as dimensões da MPK são compostas por conceitos nebulosos e acrescenta que tal subjetividade traz imprecisão aos modelos de portfólio.

Outras críticas aos modelos de portfólio de compras: (a) as dimensões da matriz são complexas [15] e os fatores escolhidos para avaliação das dimensões podem não representálas adequadamente [10]; (b) há uma baixa discriminação das categorias classificadas dentro de um mesmo quadrante [19], relacionada ao fato de a escala de medição dos eixos da matriz de portfólio representar apenas dois valores - "alto" e "baixo" [20]; (c) por se basear em uma perspectiva estritamente econômica, vantagens competitivas sustentáveis, decorrentes de relacionamentos colaborativos de longo prazo entre a empresa e seus fornecedores, por exemplo, não são consideradas [21].

Em [22], os autores fazem um estudo aprofundado sobre as empresas holandesas com o objetivo de identificar aspectos práticos da utilização MPC. Um ponto importante da aplicação dos portfólios de compra, que recebe pouca atenção na literatura especializada, diz respeito à escolha da unidade de análise utilizada na construção dos modelos. Em [9] e [23] classifica-se itens da indústria de construção, agrupando-se os materiais com o mercado fornecedor comum. Em [12], os autores sugerem que os produtos de uma mesma categoria tenham avaliações similares, quanto ao risco de suprimento. Há trabalhos em que se propõe modelos de portfólio focados nas relações específicas entre a empresa compradora e seus fornecedores ([15] [10] [24]) e, até mesmo, analisando compras específicas de uma determinada empresa [25]. Segundo [3], o padrão de categorização e taxonomia usado para classificar os produtos influenciam no posicionamento desses produtos no portfólio de compras e, consequentemente, nos direcionadores estratégicos recomendados. Neste trabalho, a unidade de análise a ser adotada é a categoria do material, que consiste em um agrupamento de itens similares, quanto a sua aplicação nas unidades organizacionais [26], e que podem ser adquiridos de um mesmo conjunto de fornecedores ou segmento de mercado.

Entende-se que o posicionamento dos produtos nos quadrantes dos portfólios de compra e, consequentemente, as estratégias escolhidas para cada categoria, são sensíveis à forma adotada para definição das categorias. Em [27], o autor estuda a análise de dispêndios e recomenda a adoção de um padrão hierárquico de classes para categorizar produtos e serviços, tal como o UNSPSC (United Nations Standard Products and Services Code). Esse padrão estabelece um código único de 8 dígitos para cada produto e serviço, no formato SSFF-CC-DD, onde SS representa o segmento, FF a família, CC a classe e DD o item. Dentre as vantagens apresentadas pelos autores para a adoção desse padrão, destacam-se: (i) facilidade de análises, em diferentes níveis, utilizando-se os códigos; (ii) todos os elementos de um produto são definidos em algum nível da hierarquia; (iii) grande abrangência de produtos e serviços já registrados.

Segundo [28], os termos compra e aquisição são muito confundidos, embora sejam diferentes no escopo. Compra, geralmente, se refere às compras reais de materiais e às 
atividades associadas ao processo de compra. Já aquisição tem um significado mais amplo e inclui a compra, transporte, armazenagem e recibo dos materiais internos, sendo parte de uma Gestão Estratégica de Suprimentos (GES) que por sua vez é parte da administração das organizações. Por definição, comprar materiais e serviços implica em que estes devem ter qualidade adequada, quantidade certa, no tempo certo e de fonte fornecedora certa. Este é o cerne da função Compras. A partir dos anos 70, o foco das empresas passou a ser a satisfação dos clientes e os processos padronizados existentes deixaram de ser eficientes com os departamentos de compras se limitando apenas a processar pedidos, com poucas oportunidades de atuarem no processo decisório da Compra, seleção de fornecedores e outros. Para [29], "fazer ou comprar” é uma decisão estratégica e traz implicações para a estratégia corporativa da organização como um todo" ver [30].

Em [31], apresenta-se um modelo de portfólio para a definição de estratégias de suprimentos na atividade de compras com base nas dimensões "importância estratégica da compra” e "complexidade do mercado fornecedor". O autor propõe que as categorias de itens adquiridos por uma empresa sejam avaliadas e classificadas nestes dois aspectos, resultando na MPK. O objetivo principal da MPK é proporcionar uma ferramenta para elaboração de estratégias capazes de minimizar os riscos de suprimento e potencializar o poder de compra das organizações. Em [16], se adota nomenclatura diferente para as dimensões da MPK, referindo-se a elas como "Impacto no Lucro" e "Risco de Suprimento". A importância estratégica da compra pode ser medida por meio dos custos dos produtos e serviços adquiridos, valor agregado e impacto no lucro gerado pelo produto ou serviço. A complexidade do mercado fornecedor é determinada pelo número de fornecedores de um dado produto, condições de concentração de mercado (e.g. monopólio e oligopólio), o ritmo do avanço tecnológico, barreiras de entrada, custos logísticos e complexidade [31].

Na MPK, cada produto, serviço ou agrupamento de produtos ou serviços similares é avaliado por critérios de medição, definidos para cada dimensão [9]. Um dos modelos de GES mais difundidos é o da matriz de posicionamento de materiais (MPM), baseado na MPK. Esse modelo apoia a administração de empresa, na seleção das estratégias de compras mais apropriadas para diferentes tipos de produtos e serviços, otimizando a relação entre impactos financeiros e risco (como falta de materiais, descontinuidades, atrasos, rompimento de contratos, entre outros). Através da MPM aplicada nas empresas, pode-se classificar os itens de compra em quatro quadrantes, conforme ilustrado na Figura 1, a seguir.

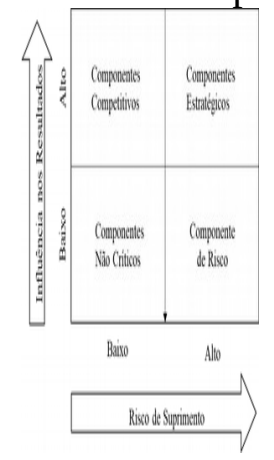

Figura 1 - MPM (Fonte: Adaptado de [32])

O impacto sobre o resultado desta aplicação, pode ser determinado por fatores como custo, atendimento, tecnologia e qualidade. O risco da oferta pode ser determinado por fatores como poder de compra, número de fornecedores, disponibilidade de reservas alternativas, estabilidade de cada fornecedor, custo de substituição, dentre outros. Com os materiais (ou grupos de materiais) classificados, uma série de políticas diferenciadas é definida para cada grupo.

A Figura 2 apresenta as políticas ou modalidades de compra e relacionamento mais 
adotadas por empresas em relação a seus fornecedores, em função do posicionamento que os produtos comprados têm na Matriz de Kraljic/MPM [33].

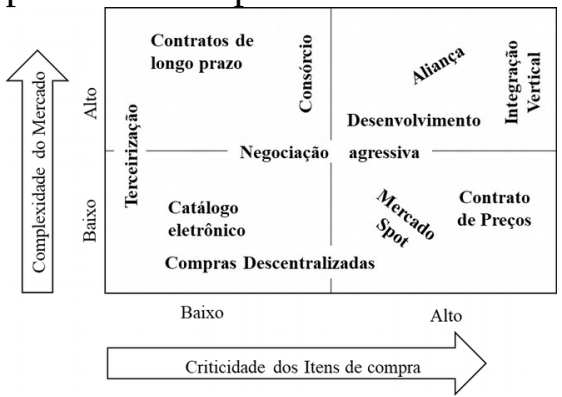

Figura 2 - Modalidades de compras Kraljic/MPM (Fonte: Adaptado de [33])

Os materiais ou serviços posicionados no quadrante dos produtos estratégicos não só têm grande impacto no resultado final de lucros, mas também podem ser difíceis de se obter ou os fornecedores de difícil substituição. Nesse caso, práticas de aliança e parceria entre clientes e fornecedores são recomendadas. Como esses produtos contêm um certo risco de oferta, é importante estabelecer relacionamentos de longo prazo ou parcerias estratégicas com seus fornecedores. Pode-se usar também práticas de desenvolvimento de novos fornecedores, bem como desenvolver materiais que possam substituí-los e/ou eliminar a utilização dos mesmos. O grupo de ‘produtos competitivos' (ou de alavancagem) representa uma grande parte do impacto que a compra tem sobre o resultado financeiro da empresa, mas por outro lado, há abundância de fornecedores, podendo-se incrementar a competição entre eles, afinal, o comprador sempre pode escolher outro fornecedor. Os produtos competitivos requerem contratos centrais com termos e condições gerais. Em alguns casos, entretanto, estratégias de concentrar as compras em um ou dois fornecedores pode representar ganhos de escala significativos e consequente redução de custos. Essa parece ser uma tendência em grandes empresas em relação a determinados fornecedores.

Os ‘produtos de risco’ (ou restrições/gargalos) são indesejados, de uma forma geral. A interrupção de seu fornecimento pode gerar sérias conseqüências para a empresa, pois este se associa a um alto impacto financeiro. A empresa precisa garantir o fornecimento ou estar protegida contra a falta deles. Isso a obriga ter estoque extra, restrições e/ou contratos com fornecedores com grandes penalidades no caso de violação ou quebra de contrato.

Os 'produtos não críticos' (ou de rotina) são tipicamente materiais de escritório, de alimentação, componentes de pequeno valor agregado etc. Esses produtos vêm sendo, cada vez mais, adquiridos através de processos automatizados de compras e ou reposição. As empresas podem ter um número limitado de fornecedores para uma ampla gama de produtos, permitindo a racionalização do tratamento administrativo e negociações com descontos. $\mathrm{O}$ resultado da implementação da abordagem MPM, com o posicionamento de cada grupo de materiais, é função do tipo de atividade desenvolvida por cada empresa. A caracterização do posicionamento dos itens de compra de uma empresa, associado aos critérios de seleção são os itens básicos para suportar uma decisão qualificada de fornecimento. Diante do exposto acima, pode-se afirmar que a atividade de compras é uma função relevante para a competitividade das organizações, o que pode ser constatado no exemplo de aplicação do modelo proposto, na seção 3, mais adiante.

\subsection{CONJUNTOS FUZZY E SISTEMAS DE INFERÊNCIA FUZZY (FIS)}

O conceito de variáveis linguísticas é muito útil ao lidarmos com situações complexas e/ou difíceis de serem descritas em termos objetivos e quantitativos [34]. Há registros de aplicações bem-sucedidas em diversos campos do conhecimento, tais como inteligência artificial, ciências da computação, engenharia, teoria da decisão, sistemas especialistas, lógica, administração e gestão, pesquisa operacional e robótica [35]. 


\section{Conjuntos Fuzzy}

A teoria de conjuntos na lógica clássica funciona de maneira booleana, isto é, um elemento pode pertencer ou não a um determinado conjunto. A estes elementos que atendem a lógica clássica dá-se o nome de crisp, que significa dicotomia ou bivalência. Na teoria dos conjuntos fuzzy, por sua vez, admitem-se diferentes graus de pertencimento ou pertinência dos elementos a um determinado conjunto. Há uma região nebulosa no limite de pertencer ou não a um conjunto. A estes elementos nomeia-se fuzzy, que significa difuso ou nebuloso.

Um número fuzzy $F$, definido em $\mathfrak{R}$, é um Número Fuzzy Triangular (NFT) se sua função de pertinência $\mu_{\mathrm{F}}(\mathrm{x}): \mathfrak{R} \rightarrow[0,1]$

Para:

$$
\mu_{F}(x)=\left\{\begin{array}{l}
\frac{x-a}{b-a}, a \leq x \leq b \\
\frac{c-x}{c-b}, b \leq x \leq c \\
0, \text { caso contrário, }
\end{array}\right.
$$

As propriedades básicas de números fuzzy triangulares ([36] [37]) são: Inversão de 1 NFT; Adição de 2 NFT; Subtração de 2 NFT; Multiplicação escalar; Multiplicação de 2 NFT; Divisão de 2 NFT.

O processo de defuzzificação é a operação final do sistema e consiste na transformação dos resultados fuzzy, obtidos do sistema de inferência, em valores crisp. A literatura apresenta cinco métodos de defuzzificação: (i) do Centro de Massa ou Centróide; (ii) da Média dos Máximos; (iii) da Média Ponderada dos Máximos; (iv) do Critério Máximo (ou Mínimo); (v) do Critério Máximo (ou Mínimo), sendo o método do centroide o mais utilizado [38], mostrado a seguir: $d f_{F}=\frac{a+b+c}{3}$

\section{Sistemas de inferência fuzzy}

Os sistemas de inferência fuzzy possuem larga aplicação no mundo real e são projetados para determinar as variáveis de saída crisp, a partir de dados de entrada também crisp, porém utilizam a lógica fuzzy no processo de inferência (tomada de decisão). São compostos por quatro blocos funcionais [39], que se encontram descritos a seguir e representados no modelo da Figura 3.

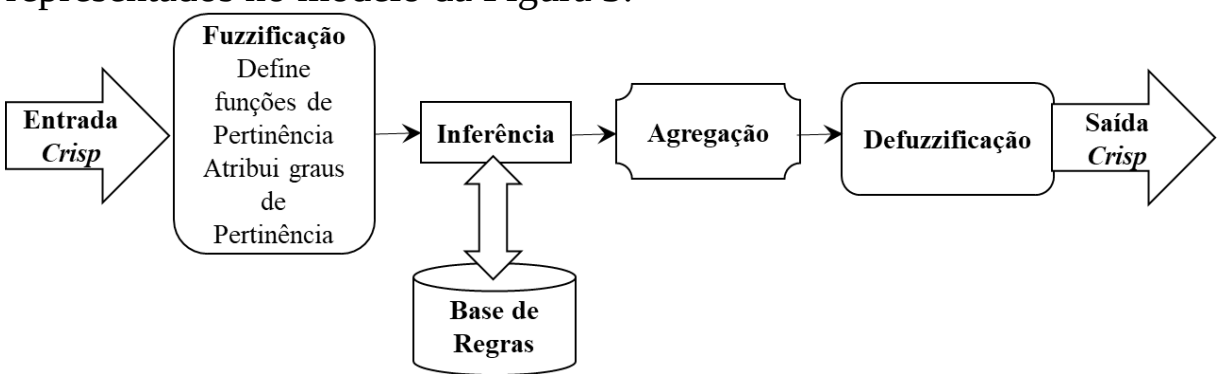

Figura 3 - Modelo esquemático de um sistema de inferência fuzzy. Adaptado de [41]

\section{MODELO DE CLASSIFICAÇÃO EM EMPRESA PÚBLICA}

Apresenta-se a seguir um estudo de caso em que se aplica o modelo estudado, em uma empresa pública do setor elétrico [30]. É importante frisar que, na ótica de [31] e [33] a classificação de materiais é o primeiro passo para o estabelecimento de uma estratégia corporativa de suprimentos. Os fatores para se classificar os materiais são: criticidade e complexidade. Cada um de estes, por serem subjetivos, dependem muito da percepção dos especialistas que respondem um questionário. $\mathrm{O}$ fator criticidade diz respeito ao risco no suprimento do produto e agrega um conjunto de critérios, aos quais os entrevistados devem atribuir pesos (dentre 1 e 7). O fator complexidade é uma simplificação da importância 
estratégica do objeto para o resultado dos negócios da empresa, também denominado de impacto nos lucros e, da mesma forma que a criticidade, agrega um conjunto de critérios, aos quais os entrevistados devem atribuir pesos (dentre 1 e 7). Após a atribuição dos pesos aos critérios procede-se a escolha do grau de intensidade (varia de 1 até 9 - usando-se métrica baseada na escala de [40] - para o atributo do critério). Desta forma, estabelece-se a contribuição do critério ao fator. Os critérios associados à criticidade são apresentados na Tabela 1.

\begin{tabular}{|l|l|l|}
\hline \multicolumn{2}{|c|}{ Critérios } & \multicolumn{1}{c|}{ Descrição } \\
\hline A1. & No $^{\text {de requisitantes }}$ & $\begin{array}{l}\text { Em virtude da ambigüidade causada pelo termo "Usuário" optou-se por } \\
\text { atribuir o peso pelo número de requisitantes do item. }\end{array}$ \\
\hline A2. & $\begin{array}{l}\text { Frequência média da compra } \\
\mathrm{N}^{0} \text { de Meses [ ] }\end{array}$ & $\begin{array}{l}\text { Caracteriza o tempo médio em que se repete a compra do item. } \\
\text { A unidade de medida é o mês }\end{array}$ \\
\hline A3. & $\begin{array}{l}\text { Grau de impacto por inexis- } \\
\text { tência do estoque. }\end{array}$ & $\begin{array}{l}\text { Caracteriza o quanto a falta do item no estoque pode prejudicar o anda- } \\
\text { mento das atividades da empresa ou de um setor da empresa. }\end{array}$ \\
\hline A4. & $\begin{array}{l}\text { Risco de interrupção do ser- } \\
\text { viço.: }\end{array}$ & $\begin{array}{l}\text { Caracteriza o peso do grau de risco no conjunto de critérios, de inter- } \\
\text { romper o serviço/ou serviços prestados pela empresa, caso ocorra falha } \\
\text { no produto. }\end{array}$ \\
\hline A5. & $\begin{array}{l}\text { Tempo de vida útil do pro- } \\
\text { duto: }\end{array}$ & $\begin{array}{l}\text { Estipula qual o peso do tempo de vida útil do produto para o conjunto } \\
\text { de critérios. }\end{array}$ \\
\hline A6. & $\begin{array}{l}\text { Tempo de Recuperação de } \\
\text { falhas: }\end{array}$ & $\begin{array}{l}\text { Caracteriza o peso do processo de recuperar a produção de um bem ou } \\
\text { serviço no conjunto de critérios. }\end{array}$ \\
\hline A7. & Frequência de Manutenção: & $\begin{array}{l}\text { Caracteriza o peso da freqüência de manutenção de um produto no con- } \\
\text { junto de critérios. }\end{array}$ \\
\hline
\end{tabular}

Tabela 1 - Critérios associados à criticidade: pesos e descrição dos materiais [30]

Convenciona-se que a mensuração da intensidade dos atributos deve ser realizada após o registro do peso dos critérios (A1 a A7). A intensidade está configurada em três níveis: baixa, média e alta. Cada nível é estruturado em três subníveis. Os critérios e atributos, ajustados após discussão com os especialistas, são considerados por estes, como autoexplicativos. Também são desenvolvidas as mesmas métricas para o critério complexidade e uma breve descrição é apresentada na Tabela 2.

\begin{tabular}{|c|c|c|}
\hline \multicolumn{2}{|r|}{ Critérios } & Descrição \\
\hline B1. & $\begin{array}{l}\mathrm{N}^{\mathrm{os}} \text { de fornecedores: nacional, } \\
\text { estrangeiro com sede no país; } \\
\text { estrangeiro sem sede no país }\end{array}$ & $\begin{array}{l}\text { Quanto maior o número de fornecedores atuais e/ou potenciais me- } \\
\text { nor a complexidade do mercado }\end{array}$ \\
\hline B2. & Valor unitário: & $\begin{array}{l}\text { Quanto maior o peso do atributo maior o valor unitário do material } \\
\text { que está sendo adquirido. Tanto maior será a complexidade da com- } \\
\text { pra quanto maior for o valor do material. A unidade de medida é } \\
\text { monetária R\$ ou U\$ }\end{array}$ \\
\hline B3. & $\begin{array}{l}\text { Quantidade média adquirida } \\
\text { por ano: (compras isoladas) } \\
\text { (Q: }\end{array}$ & $\begin{array}{l}\text { Caracteriza os lotes de compra ou o volume médio de itens compra- } \\
\text { dos. }\end{array}$ \\
\hline B4. & Especificação técnica: & $\begin{array}{l}\text { Caracteriza o peso do nível de especificação exigida para a aquisi- } \\
\text { ção do item no conjunto de critérios. }\end{array}$ \\
\hline B5. & $\begin{array}{l}\text { Serviço Técnico oferecido } \\
\text { pelo fornecedor.: }\end{array}$ & $\begin{array}{l}\text { Estipula qual o peso do nível de serviço prestado pelo fornecedor } \\
\text { para o item no conjunto de critérios. }\end{array}$ \\
\hline
\end{tabular}

Tabela 2 - Critérios associados à complexidade: pesos e descrição dos materiais [30]

Por sua vez, convenciona-se que a mensuração da intensidade dos atributos deve ser realizada após o registro do peso dos critérios de complexidade (B1 a B5). A intensidade está configurada em três níveis: baixa, média e alta. Cada nível é estruturado em três subníveis. A seguir, são mostradas as principais características do modelo de Lógica fuzzy, implementado no Matlab [41]. Os termos lingüísticos usados na implementação correspondem a duas variáveis: criticidade e complexidade, cada uma delas formadas por 7 e 5 variáveis, respectivamente. Cada uma delas é formada por uma partição fuzzy, composta pelos seguintes valores lingüísticos: “muito ruim” (MR), "ruim” (R), “médio” (M), “bom” (B) e 
“muito bom” (MB). Cada um destes valores lingüísticos é formado por uma função triangular, como mostrada a seguir. Os parâmetros da função triangular são a, b, e c; quando a variável x é igual a b e a função triangular atinge seu valor máximo.

$$
f(x ; a, b, c)=\max \left(\min \left(\frac{x-a}{b-a}, \frac{c-x}{c-b}\right), 0\right)
$$

A disposição da partição fuzzy ou nebulosa é dada em um domínio de 1 a 9, com os valores lingüísticos de MR sendo $[1,1,3]$ e os de $\operatorname{MB}[7,9,9]$, o que mostra triângulosretângulos no início e no fim do domínio da partição, como mostrado na Figura 4.

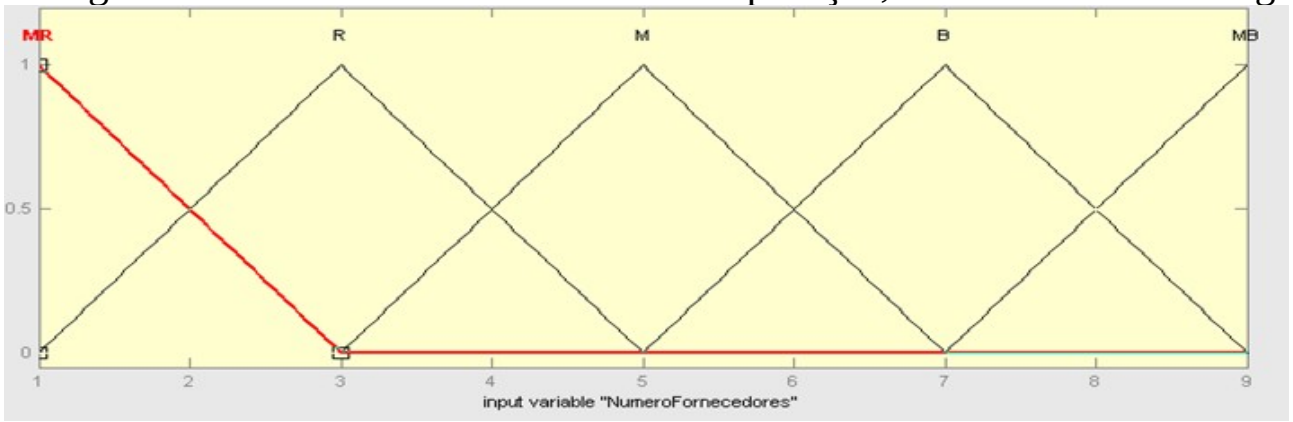

Figura 4 - Participação nebulosa dos termos lingüísticos [41]

As regras lógicas correspondem a 35 processos de inferência do tipo if-then, considerando-se cada um dos 7 tipos de pesos diferentes, no caso da criticidade. Estes pesos correspondem, à média fornecida pelos especialistas. No caso da complexidade, as regras são formadas em 25 processos de inferência e cada uma delas se apresenta com seu respectivo peso. Na Figura 5 são mostradas as sentenças lógicas, como um exemplo.

\begin{tabular}{|c|c|}
\hline 1. If (Numerofornecedores is MR) then (Criticidade is MR) (0.75) & $\Delta$ \\
\hline 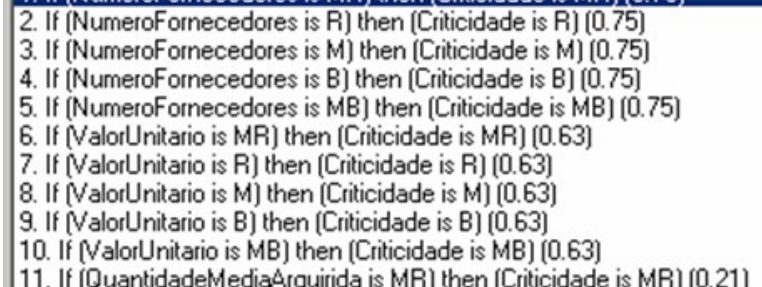 & 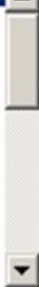 \\
\hline
\end{tabular}

Figura 5 - Procedimentos lógicos de if_then para definir o item de complexidade [41]

O procedimento de agregação é definido como a função máximo, que corresponde a uma operação de união fuzzy ou nebulosa dos conjuntos. Desta operação resulta um conjunto fuzzy que, através do uso do centróide, encontra-se um valor crisp como valor de número da agregação. A seguir, é mostrado na Figura 6, a título de exemplo, o processo de agregação e defuzzificação, aplicado no exemplo ilustrativo do estudo de caso.

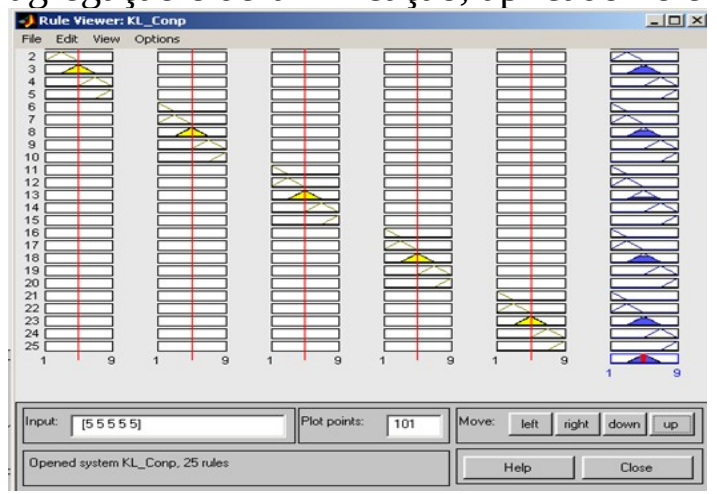

Figura 6 - Agregação e defuzzificação na complexidade [41]

Os procedimentos estruturados apresentados anteriormente proporcionam uma 
classificação de materiais.

\section{RESULTADOS COMPUTACIONAIS}

Uma representação dos resultados produzidos sobre um grupo de materiais com as informações dadas pelo grupo de profissionais entrevistados, no estudo de caso, é apresentada na Figura 7 e Tabela 3.

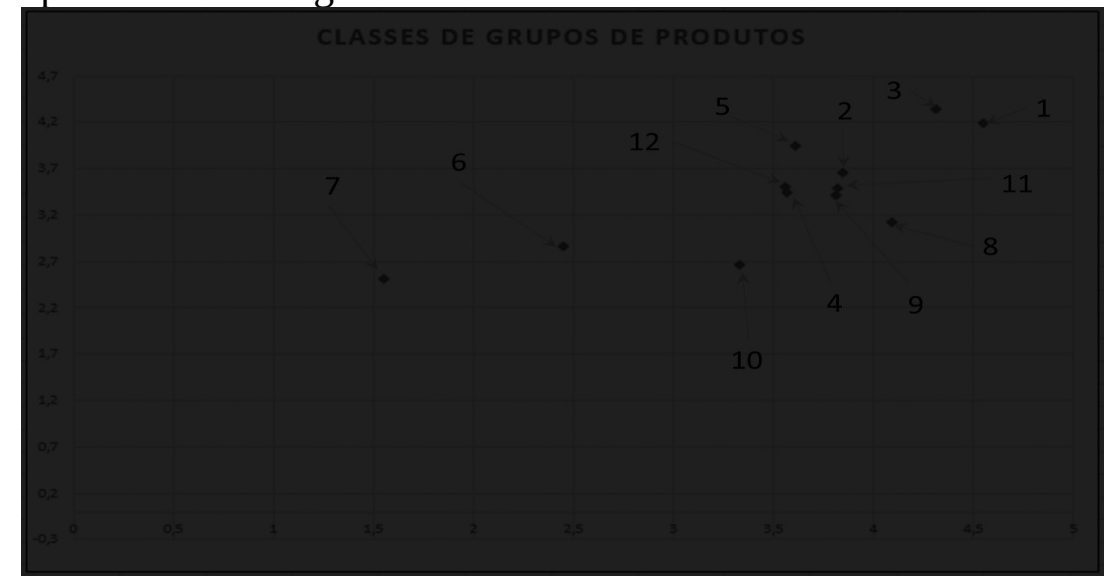

Figura 7 - Classificação de grupos de produtos por criticidade e complexidade - todos os profissionais [30]

\begin{tabular}{|r|l|r|r|r|}
\hline & \multicolumn{1}{|c|}{ Produto/código } & Cod. & \multicolumn{1}{c|}{ Criticidade } & Complexidade \\
\hline 1 & Autotransformador & 5740 & 4,55 & 4,19 \\
\hline 2 & Transformadores de corrente & 5767 & 3,85 & 3,65 \\
\hline 3 & Reatores & 5726 & 4,31 & 4,34 \\
\hline 4 & Disjuntores & 5818 & 3,57 & 3,44 \\
\hline 5 & Banco de capacitores & 5801 & 3,61 & 3,95 \\
\hline 6 & Painéis de controle & 6373 & 2,45 & 2,86 \\
\hline 7 & Comput, periféricos, suprimentos & 7460 & 1,55 & 2,52 \\
\hline 8 & Para-raios & 5808 & 4,09 & 3,13 \\
\hline 9 & Seccionadores & 5773 & 3,81 & 3,41 \\
\hline 10 & Cabos elétricos & 5773 & 3,33 & 2,67 \\
\hline 11 & Isolador disco & 5765 & 3,82 & 3,49 \\
\hline 12 & Amortecedor-espaçador & 5777 & 3,56 & 3,51 \\
\hline
\end{tabular}

Tabela 3 - Classificação de grupos de produtos por criticidade e complexidade [30]

Para efeito de representação, a ponderação estabeleceu-se como referência para a divisão dos quadrantes, os eixos horizontais e verticais. A representação mostra que os profissionais atribuem alta criticidade a praticamente todos os itens e não identificam nenhum item como alavancador. Esta percepção do conjunto indica uma valorização do conhecimento funcional, ou seja, atribui-se maior valor ao que se conhece ou se relaciona com a função desempenhada na organização. Apenas o grupo "computador" foi caracterizada como itens rotineiros.

Uma percepção mais apurada da criticidade está associada aos profissionais que lidam diretamente com o risco de suprimento. Pela natureza das atividades, o risco maior de suprimento está associado aos equipamentos e materiais empregados em usinas, subestações e linhas de transmissão. Os profissionais que atuam na engenharia assumem um papel decisivo na seleção e especificação de equipamentos e materiais para os ativos, enquanto os da operação e manutenção têm sua atuação mais focada no dia-a-dia do monitoramento, operação e melhorias dos ativos associados ao core business da organização.

\section{CONSIDERAÇÕES FINAIS}

O presente artigo tem como foco o processo de classificação de materiais, no qual a 
empresa inicia seu processo de agregação de valor, no que diz respeito ao processo de compras, cujas implicações ocorrem em seu lucro, a curto e longo prazo. Com os materiais (ou grupos de materiais) classificados, uma série de políticas diferenciadas podem ser definidas para cada grupo. Materiais classificados como estratégicos são tratados de forma diferenciada que os materiais rotineiros.

A metodologia proposta de classificação mostrou-se viável e apropriada para o posicionamento dos grupos de materiais a serem adquiridos. Sua simplicidade de aplicação com o uso de termos linguísticos, através de levantamento de informações em questionários, a torna uma ferramenta que pode atrair a percepção de cada profissional das diversas áreas da empresa, para cada item ou grupo de itens classificados. Os profissionais, que podem melhor contribuir para tais critérios de avaliação e classificação, são os pertencentes aos setores de: compras, manutenção e reparo.

A metodologia proposta é melhor aplicada, quando se formam grupos a serem avaliados, uma vez que muitas empresas possuem milhares de itens de materiais, o que tornaria inviável o levantamento dos critérios de criticidade e complexidade para cada um deles. A etapa de validação do portfólio de compras com os líderes e especialistas das organizações é fundamental, pois retroalimenta a base de conhecimento, identificando pontos de melhorias e corrigindo inconsistências na modelagem do problema.

\section{REFERÊNCIAS BIBLIOGRÁFICAS}

[1] BRIGGS, P. Vendor assessment for partners in supply. European Journal of Purchasing \& Supply Management, v. 1, p. 49-59, 1994.

[2] CHOI, T.Y.; HARTLEY, J.L. An exploration of supplier selection practices across the supply chain. Journal of Operations Management, v. 14, p. 333-343, 1996.

[3] LIMA, J.R.A.O.; IGNACIO, A.A.V. Modelo fuzzy para posicionamento de produtos na matriz de portfólio de compras de Kraljic: Um estudo de caso. In: XXII Simpósio de Engenharia de Produção. 2015. Bauru. Anais [...]. Bauru: XII SIMPEP, 2015.

[4] MARKOWITZ, H. Portfolio selection. Journal of Finance, v. 7, n. 1, p. 77-91, 1952.

[5] TURNBULL, P.W. A Review of Portfolio Planning Models for Industrial Marketing and Purchasing Management. European Journal of Marketing, v. 24, n. 3, p. 7-22, 1990.

[6] ANDERSSON, S.; SERVAIS, P. Combining industrial buyer and seller strategies for international supply and marketing management. European Business Review, v. 22, n. 1, p. 64-81, 2010.

[7] ZADEH, L.A.; Fuzzy sets. Information and control, 1965.

[8] ZADEH, L.A.; The Concept of a Linguistic Variable and Its Application to Approyimate Reasoning. Working paper, UC Berkeley, 1973.

[9] ARANTES, A.; FERREIRA, L.M.D.F.; KHARLAMOV, A.A. Application of a Purchasing Portfolio Model in a Construction Company in Two Distinct Markets. Journal of Management in Engineering, v. 30, n. 5, 2014.

[10] NELLORE, R.; SÖDERQUIST, K. Portfolio approaches to procurement: Analyzing the missing link to specifications. Long range planning, v. 33, n. 2, p. 245-267, 2000.

[11] CANIËLS, M.C.J.; GELDERMAN, C.J. Power and interdependence in buyer supplier relationships: A purchasing portfolio approach. Industrial Marketing Management, v. 36, p. 219-229, 2007. 
[12] GELDERMAN, C.J.; MAC DONALD, D.R. Application of Kraljic's Purchasing Portfolio Matrix in an Undeveloped Logistics Infrastructure: The Staatsolie Suriname Case. Journal of Transnational Management, v. 13, n. 1, p. 77-92, 2008.

[13] SEIFBARGHY, M. Measurement of supply risk and determining supply strategy, case study: A refrigerator making company. 40th International Conference on Computers and Industrial Engineering: Soft Computing Techniques for Advanced Manufacturing and Service Systems, CIE40 2010, Proceedings [...] New York: IEEE. p. 776-780, 2010.

[14] TERPEND, R.; KRAUSE, D.R.; DOOLEY, K.J. Managing buyer-supplier relationships: empirical patterns of strategy formulation in industrial purchasing. Journal of Supply Chain Management, v. 47, n. 1, p. 73-94, 2011.

[15] OLSEN, R.F.; ELLRAM, L.M. A portfolio approach to supplier relationships. Industrial Marketing Management, v. 26, n. 2, p. 101-113, 1997.

[16] GELDERMAN, C.J.; WEELE, A.J. Strategic Direction through Purchasing Portfolio Management: A Case Study. The Journal of Supply Chain Management, v. 38, n. 1, p. 30-37, 2002.

[17] DAY, G.S. Analysis for Strategic Market Decisions. St Paul: West Publishing Company, 1986.

[18] RAMSAY, J. Purchasing power. European Journal of Purchasing and Supply Management, v. 1, n. 3, p. 125-138, 1994.

[19] COX, A. Understanding buyer and supplier power: a framework for procurement and supply competence. Journal of Supply Chain Management, v. 37, n. 2, p. 8-15, 2001.

[20] PADHI, S.S.; WAGNER, S.M.; AGGARWAL, V. Positioning of commodities using the Kraljic Portfolio Matrix. Journal of Purchasing and Supply Management, v. 18, n. 1, p. 1-8, 2012.

[21] WAGNER, S.M.; JOHNSON, J.L. Configuring and managing strategic supplier portfolios. Industrial Marketing Management, v. 33, n. 8, p. 717-730, 2004.

[22] GELDERMAN, C.J.; WEELE, A.J. Handling measurement issues and strategic directions in Kraljic's purchasing portfolio model. Journal of Purchasing and Supply Management, v. 9, n. 5-6, p. 207-216, 2003.

[23] FERREIRA, L.M.D.F.; ARANTES, A.; KHARLAMOV, A.A. Development of a purchasing portfolio model for the construction industry: an empirical study. Production Planning \& Control, v. 26, n. 5, p. 1-16, 2014.

[24] BOHME, T.; CHILDERHOUSE, P.; DEAKINS, E.; CORNER, J.(*) Balancing power and dependency in buyer-supplier relationships. International Journal of Electronic Customer Relationship Management, v. 2, n. 2, p. 120-139, 2008.

[25] OSIRO, L. Uso da lógica fuzzy para avaliação e desenvolvimento de fornecedores baseado em modelos de portfólio. Tese (Doutorado) - Escola de Engenharia de São Carlos da Universidade de São Paulo. São Carlos, 2013.

[26] TRAUTMANN, G.; BALS, L.; HARTMANN, E. Global sourcing in integrated network structures: The case of hybrid purchasing organizations. Journal of International Management, v. 15, n. 2, p. 194-208, 2009. 
[27] MARZIC, S.; KRNETA, P.; PAVLIC, M. Spend analysis systems. Proceedings [...]. Croatia: 37th International Convention MIPRO 2014.

[28] STOCK, J, LAMBERT, D.M. Strategic Logistics Management. Irwin, 1993.

[29] HUMPHREYS, P.K.; LO, V.H.Y.; MCIVOR, R.T. A decision support framework for strategic purchasing. Journal of Processing Materials Technology. v. 107, n.1-3, p. 353-362, 2000.

[30] IGNACIO, A.A.V.; FERNANDES, E.; SAMPAIO, L.M.D.; ARAÚJO, R.S.B. O papel do custo total de propriedade (TCO) no gerenciamento da cadeia de suprimentos. Anais[...]XIII SIMPEP. Bauru, SP/BR, 2006.

[31] KRALJIC, P. Purchasing must become supply management. Harvard Business Review, v. 61, n. 5, p. 109-117, 1983.

[32] CAVANHA, A.O.F. Logística: Novos Modelos. Qualimark, Rio de Janeiro, 2001.

[33] DE PELLEGRIN, I.A.; BARBOSA, R.S. Caracterização do arranjo produtivo do petróleo da Bacia de Campos e a estruturação de uma rede de empresas. Rede PetroBC, Sebrae-RJ, Rio de Janeiro, 2004.

[34] ZIMMERMANN, H.-J. Fuzzy Set Theory - And its Applications. Boston; Dordrecht; London: Kluwer Academic Publishers, 1991.

[35] ZIMMERMANN, H.-J., 2001. Fuzzy Set Theory e and Its Applications, vol. 30. Springer Science \& Business Media, Norwell, Massachusetts.

[36] DENG, H. Multicriteria analysis with fuzzy pairwise comparison. International Journal of Approximate Reasoning, v. 21, n. 3, p. 215-231, 1999.

[37] WANG, T.-C.; CHEN, Y.-H. Applying fuzzy linguistic preference relations to the improvement of consistency of fuzzy AHP. Information Sciences, v. 178, n. 19, p. 3755-3765, 2008.

[38] CHOU, S.-Y., CHANG, Y.-H. A decision support system for supplier selection based on a strategy-aligned fuzzy SMART approach. Expert Systems with Applications, v. 34, n. 4, p. 2241- 2253, 2008

[39] REZAEI, J.; ORTT, R. Multi-criteria supplier segmentation using a fuzzy preference relations based AHP. European Journal of Operational Research, v. 225, n. 1, p. 75-84, 2013a.

[40] SAATY, T. Métodos de Análise Hierárquica. Rio de Janeiro, RJ: Ed. Makron, 1991.

[41] SAMPAIO, L.M.D., OLIVEIRA, M.J.F., IGNACIO, A.A.V. Lógica Nebulosa: Aplicações E Tendências. Anais[...] SPOLM2007, ISSN 2175-6295 Rio de JaneiroBrasil, 07 e 08 de novembro de 2007. 